\title{
Vocal Cord Dysfunction in Asthmatics
}

\author{
Yvonne L. McCarey, MD
}

\section{Case Presentation}

A 44 year old female with a past medical history of type 2 diabetes, hypertension, congestive heart failure, coronary artery disease, 2 strokes, 2 myocardial infarctions, questionable history of pulmonary embolus twice, bipolar disorder, schizophrenia, and multiple asthma exacerbations presented to the Emergency Department (ED) with SOB and wheezing. Her symptoms began at home earlier that day and were not improved by bronchodilators. On arrival to the ED, she was lethargic in severe respiratory distress with laboring accessory muscle use. She had only an allergy to latex. Outpatient medications included albuterol inhaler, fluticasone/salmeterol inhaler, inhaled budesonide, furosemide, and warfarin for empiric treatment of an unidentified hypercoaguable disorder. She reported having a 15 pack year smoking history including marijuana and cocaine use up to several days prior to admission, but she denied any prior alcohol or intravenous (IV) drug abuse. Family history was non contributory. Her respiratory distress prohibited a thorough review of systems on admission, but the rest of her history was obtained through family at the time of arrival. They related that she had described some chest tightness earlier.

Vitals revealed the patient to be afebrile, tachypneic (26 resp/ min) saturating $100 \%$ on room air with her other hemodynamic parameters within normal limits. Significant inspiratory and expiratory wheezing were auscultated on exam and despite treatment with magnesium sulfate, bronchodilators and IV steroids, her respiratory distress did not improve. She was emergently intubated for the $6^{\text {th }}$ time in her life. Laboratory studies were significant for a normocytic anemia with a hemoglobin $8.5 \mathrm{mg} / \mathrm{dL}$ and a urine drug screen revealing the presence of cocaine. Otherwise, she had a normal metabolic panel, coagulation studies, d-dimer, normal troponins, and no EKG changes to support an ischemic event.

\section{Hospital Course}

The patient rapidly weaned off the ventilator and was extubated within 7 hours. Notably, her peak inspiratory pressures ranged from 23-26 mm Hg. Her steroids were rapidly tapered and scheduled nebulizer treatments were regularly delivered. She was discharged home without any further complication several days after admission.

\section{Discussion}

This patient carries a diagnosis of asthma with repeated intubations in our hospital's Emergency Department resulting in multiple hospital stays per year. While it is not infrequent for a severe asthma attack to require intubation, certain features are usually present, including the observation of increased peak airway pressures once ventilated. In the case presented here, the patient's peak airway pressures were normal, intubation rapidly relieved symptoms, and the patient recovered quickly.
This scenario is consistent with Vocal Cord Dysfunction (VCD) sometimes referred to as Paradoxical Vocal Cord Motion Disorder (PVCMD), Laryngeal Dyskinesia (LD) or a host of other terms, which can occur alone or in conjunction with asthma. In an acute state this condition can closely resemble an asthma attack and may therefore go unrecognized for years leading to wasted healthcare dollars for unnecessary intubations not to mention the delayed delivery of appropriate treatment. Demographics include a 2:1 female to male predominance with $71 \%$ of cases being adults above age 18 . The disorder does affect neonates, however, though this is believed likely secondary to gastroesophagela reflux disease (GERD). ${ }^{4}$

During an attack, the vocal folds adduct during inspiration and/or expiration leading to dyspnea, wheezing, cough, chest pain and stridor. Once thought to be psychogenic, evidence is growing to support mechanisms of glottic hypersensitivity secondary to various stimuli including GERD, chemical irritants, upper respiratory infection, and exercise. Altered autonomic balance, stimulation of the sensory nerve endings in the upper or lower respiratory tract, hyperventilation and hypocalcemia are also thought to play a role. ${ }^{2}$ It is important to rule out neurologic causes of VCD including brainstem compression, stroke, myasthenia gravis, amyotrophic lateral scerlosis, and focal dystonias. ${ }^{4}$

Clinical features distinguishing the disorder from asthma include irritant, exercise, and anxiety induced symptoms especially those refractory to usual asthma treatments. Lack of nocturnal awakenings and sputum may also be important clues. In addition, wheezing heard loudest over the larynx or larger airways, a normal alveolar-arterial gradient, and normal oxygen saturation, as in this patient, may help distinguish between the two. Recently, however, several cases of VCD have been observed in the setting of hypoxemia. ${ }^{2}$ Laryngoscopy remains the gold standard of diagnosis. During an attack of VCD, laryngoscopy will reveal adducted vocal cords. However, during an asthma attack, the vocal cords will not be adducted. Pulmonary function tests may be normal when asymptomatic but will show a flattening of the inspiratory and/or expiratory limb while affected. Unfortunately, methacholine challenge can induce VCD symptoms and cannot reliably be used to distinguish between asthma an vocal cord dysfunction. ${ }^{1,4}$

The similarity of symptoms and the difficulty distinguishing between VCD and asthma poses a challenge for clinicians responding to a patient in severe respiratory distress as presented above. In the acute setting, does the clinician intubate the patient, despite the increased chance of ventilator associated pneumonia and likely prolonged hospital stay? Or does the clinician risk the possible consequences of not intubating a severe asthma attack? It is likely that most clinicians would stay the conservative route 
of intubating the patient rather than attempting to discern the two entities and risking disastrous consequences.

Once VCD is adequately diagnosed several treatments can be used, even during an acute setting. The anxiety created by the patient hearing their own wheezing can be minimized by reassurance and judicious use of benzodiazepines. Heliox is the non-commercial name for a unique mixture of helium and oxygen that reduces air density therefore reducing turbulence in the airway, and in turn reducing the work of breathing and possibly relieving symptoms. ${ }^{4}$ Similarly, using a mask with a one-way pressure valve increases resistance to inspiration which will reduce stridorous sounds and diminish the patient's distress. Continuous positive airway pressure (CPAP) presumably improves symptoms by slowing expiratory flow and increasing lung volume resulting in a more open glottis. Inhaled anticholinergics, likely through a vagally mediated mechanism have been shown to prevent exercise induced symptoms in case reports only. Interestingly, botulinum toxin type A has also been reported as useful treatment to prevent attacks. ${ }^{2}$

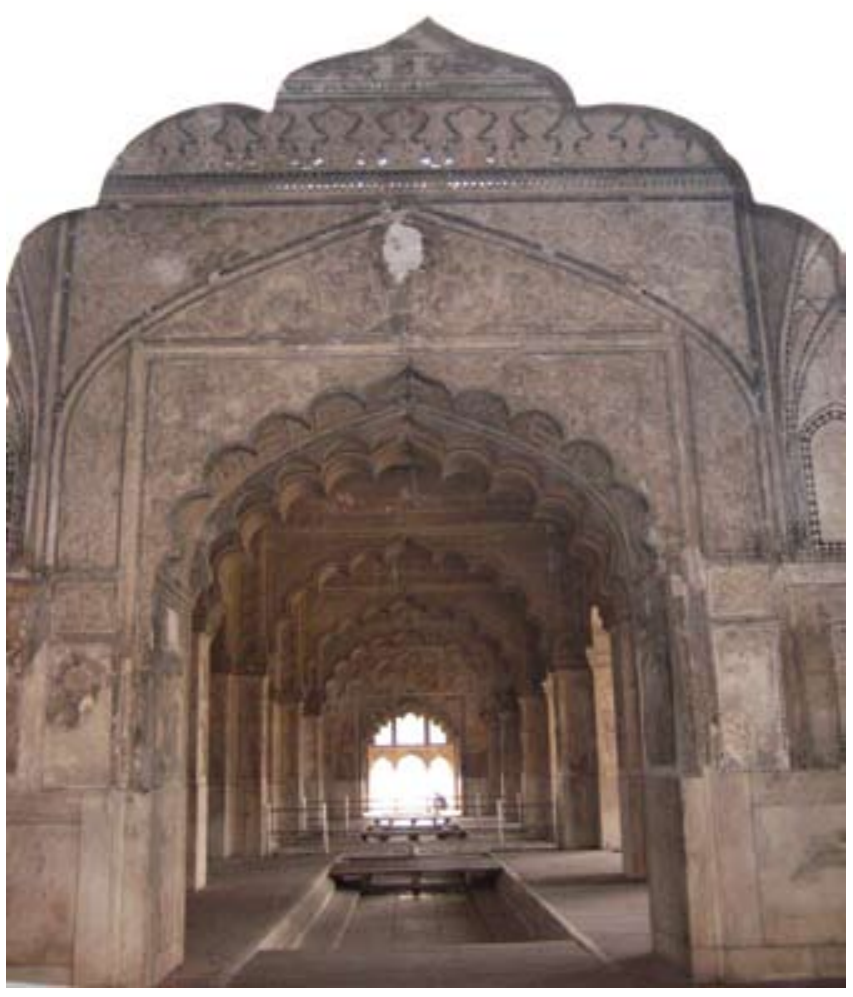

The aforementioned techniques may be utilized in the treatment of patients strongly suspected to suffer from VCD with the objective of avoiding repeated intubation and the consequences of steroid over-use. Many patients will improve with proper treatment. Long term treatment aimed at preventing attacks involves regular follow up with ENT, Pulmonary, Psychiatry and importantly a speech therapist to optimize control of the laryngeal muscles and proper breathing techniques.

\section{References}

1. Guss J, Mirza N. Methacholine Challenge Testing in the Diagnosis of Paradoxical Vocal Fold Motion. Laryngoscope. 2006;116: 1558-1561.

2. Ibrahim WH, Gheriani HA, Almohamed AA, Raza T. Paradoxical vocal cord motion disorder: past, present and future. PMJ. 2007;83: 164-172.

3. Lawrence S. Laryngeal dyskinesia: An under-recognized condition. Emergency Medicine Australia. 2007; 19: 96-104.

4. Morris MJ, Col MC, Allan PF, MAJ MC, Perkins PJ. Vocal Cord Dysfunction: Etiologies and Treatment. Clinical Pulmonary Medicine. 2006;13(2):73-86.
Corridors

Photo courtesy of

Neilanjan Nandi, MD 